\title{
VIRTUAL REALITY: THE WAY AHEAD IN INDUSTRIAL SAFETY
}

\author{
Konstantinos Loupos \\ Institute of Communication and \\ Computer Systems (ICCS) \\ 9 Iroon Polytexneiou Street \\ 15773, Athens, \\ Greece
}

\author{
Panagiotis Psonis \\ Institute of Communication and \\ Computer Systems (ICCS) \\ 9 Iroon Polytexneiou Street \\ 15773, Athens, \\ Greece
}

\author{
Dr Angelos Amditis \\ Institute of Communication and \\ Computer Systems (ICCS) \\ 9 Iroon Polytexneiou Street \\ 15773, Athens, \\ Greece
}

\section{KEYWORDS}

Virtual Reality, Virtual Environment, Industrial Safety, Simulation.

\begin{abstract}
This paper focuses on the application of Virtual Reality (VR) technologies and Virtual Environments (VEs) on the industrial field regarding safety. The issues of the industrial field are initially presented, focusing on the safety tasks currently performed in the sector. The Virtual Reality technology's innovative character is then presented, focusing on how this technology can by itself aid the safety processes or in combination with other novel technologies. The latter approach provides significant aids to methodologies that are currently used towards industrial safety and the reduction of accidents. Under this scope, this paper will refer to training, safety management, risk assessment and accident investigation as the four main safety actions currently performed in industrial fields. The paper closes by presenting in general the applicability and innovative character of VR applied to the aforementioned four safety actions as well as presenting a concrete use case example.
\end{abstract}

\section{INTRODUCTION}

Significant amounts of resources are nowadays being spent from the industrial sector in order to reduce the causes and finally actual industrial accidents that if occurred could cause property damages, human injuries and even fatalities. Therefore industrial safety can be characterized of particular importance especially when talking about hazardous material environments (chemical, petrochemical, nuclear, etc). There are always several reasons for an accident to occur; however, it may need some thought and help to figure out what has gone wrong. Once the reason has been found though, it is possible to ensure future incidents' prevention. Some actions that can be mentioned inside the framework of industrial safety are those of training, risk assessment, accident investigation and safety management. These actions can include involvement of the actual plant operators, plant managers, emergency teams or control room operators. Additionally these actions can provide particular assistance to plant managers to define the impact of certain decisions on individual (or group of) operators' work.

\section{The Industrial Situation}

There are a series of safety actions currently executed in industrial plants regarding Risk Analysis, Accident Investigation, Safety Management and Training. It can be said that the Risk Analysis and Accident Investigation can be divided into Hazard Identification, Consequence Assessment, and Accident Investigation and Accident Modelling. On the other hand, Safety Management can be defined as the control of risks that have been identified through risk assessment and ongoing learning from incidents/accidents and is a more complicated task including audit, accident prevention and mitigation, and emergency response. Furthermore, a training system involves the design of curriculum, training materials and facilities (hardware, software, simulators, instructor facilities, courseware for students and instructors) aiming at having trainees develop knowledge, skills and behavioural practices to perform their work, meeting operational goals (Childs, 1999).

What have been described above are the related industrial safety actions that are usually performed in order to increase industrial safety. As can be seen these are quite complicated and time consuming tasks that up to now (with minor exceptions) have been performed requiring strong human intervention. Later in this publication, description of the issues currently present during the execution phase of these processes has been included.

\section{Issues In Performing Safety Activities}

Some of the issues that have been raised as significant obstacles and needs during the execution of the aforementioned safety actions can be summarized below:

- Need for support of training and test of resources with regard to minor/medium emergency response scenarios.

- Representing shared and mutually know tasks and norms / good practice.

- Coordinating between the safety management functions.

- Comparing results against "best practice" instead of standards. 
- Lack of training of personnel.

- Insufficient awareness of risks and consequences among staff.

- Training involving real time simulation: fire fighting simulation; emergency response training.

- Support of accident analysis and what-if analysis.

- Need for simulating accident scenario with simulation of static and dynamic processes.

- Time constraints.

\section{VIRTUAL REALITY AS AN ENABLING TECHNOLOGY}

Concerning the identified aforementioned needs, various systems can be designed supporting a number of technologies and scientific fields from Human factors related issues up to technologies like Communications, networking, visualization, simulation, Human Machine Interfaces and others. Amongst them, one of the most important technologies to be used is Virtual reality. Recently VR and related tools have started to be used, mainly on a research level with promising results raising the interest of industry, governmental bodies and the society.

Virtual Reality technologies as a rapidly evolving and diversifying field can be regarded as one of the keytechnologies regarding visualization and is currently used in a wide area of applications such as archaeology, entertainment, architecture, automotive, industry etc. Its increasing usage in various fields has not only aided in major improvements but it has also contributed in the identification of issues and needs in different application domains. Additionally the usage of VR technologies has been proven ideal as an effective design and training tool thanks to the immersiveness (the sense of "being there") and interactivity features that it supports.

\section{Technology Insight}

The importance of Virtual Reality in systems promoting industrial safety can easily be seen if we see VR as an integration point for the technologies and equipment used. On the one hand, the VR system needs to interface with the given hardware including projection systems, tracked devices etc. in order to interact with the users. On the other, lies the application level in which the VR system is responsible for handling the $3 \mathrm{D}$ data and coordinating a real-time virtual environment, implementing the logic and behaviour of the elements constituting the virtual world and more.

The innovative character of VR in the specific field stems from the fact that it can effectively address the issues pointed out in the previous section with a low cost. The problem of simulating dynamic accident scenarios in which participants can be trained for emergency responses and enhance their awareness of risks and consequences is reduced to the task of modelling the virtual environment. From that point on the VR system can recreate the environment and allow the users to interact with it freely. A key element, however, in the success of such a system especially when time constraints are applied and evaluated, is its immersiveness. This relies greatly on the input and display technologies used.

One of the characterizing features of virtual reality is stereoscopic vision. By feeding each eye of the viewer with a different image the spectator is able to better perceive the dimension of depth thus enhancing the system's realism. A serious drawback of this technology is the need for specific projection systems (polarized projection, laser projection etc.) that usually occupy large spaces and special glasses that need to be worn by the user and may prove cumbersome. Recent advances, however, have brought forward a new breed of autostereoscopic monitors that occupy the same size as normal TFT panels and produce stereoscopic images without the need of any extra equipment. The ease of use and installation will naturally improve the overall experience and allow for further penetration of VR technologies into industrial environments. Further to this, however, one can easily imagine exploiting such technology in the form of VR-enabled handheld PCs. The applications of such a device in real-life field operations are endless since the operators will be able to receive real-time $3 \mathrm{~d}$ visualizations of information otherwise impossible to perceive (e.g. the temperature of a component). This will essentially lead to more efficient on-site decision making and lead to safer working environments.

On the other hand, equally important are the interaction devices used to manipulate the virtual world. Providing the user with natural and intuitive means of performing familiar activities is a crucial point towards achieving a truly immersive environment. Advances in tracking technologies (magnetic, optical etc.) have offered a lot to this goal. A wide variety of devices can be found today at affordable costs that allow for head, hands or even whole body tracking. Through such equipment (3D mice, data gloves etc.) it is possible to enhance the user's experience by allowing him/her to employ the same body movements as in real life in order to perform a specific activity in the virtual world. For example, using a pair of data gloves, the user could grab and rotate a virtual wheel as he/she would do in reality. In the same manner he/she could press a button by virtually touching it. Moreover, by tracking the body and/or head position of the user it is possible to update on the fly the viewing perspective of the virtual environment thus creating the illusion that the screen is actually a window to the real world. As research in this field progresses, such devices have grown to become 
extremely accurate and non-invasive rendering their usage more than practical.

\section{COMBINED TECHNOLOGIES INNOVATION}

VR technologies are geared towards creating realistic and immersive virtual environments with which users are allowed to interact freely. However, for all but the simplest applications, this is not enough. A simulation, especially one aimed at industrial safety, will require collaboration with a large range of external applications in order to implement features such as environmental rules or scenario logic. Apart from that, it is important to seek for alternate technologies that can be combined with VR and possibly expand its effectiveness or fields of application.

\section{External Simulators}

When dealing with training, it is usually the case that we simply require from the trainees to follow a certain procedure by performing successive actions such as operating various plant components. However, when the simulation is conducted to drive risk assessment or accident investigation research it is mandatory to integrate the plant's behaviour in the virtual environment. Otherwise, it would be impossible to accurately recreate a potential accident case based on the participants' actions. Such functionality can be provided by external industrial simulators that are available in the market and allow for real-time, precise and reliable simulation of a plant's general product flow as well as individual component mechanics. On the other hand, as this type of software is designed to reproduce the "internal" operation of an industrial site, it fails to provide data regarding the consequences of an accident towards the environment. Imagine for example the case of a petrochemical plant in which a gas leakage has been identified. Although a plant simulator would identify it and update the related components accordingly (e.g. pressure drop in a pipe), no information can be produced regarding the emitting of gas to the environment.

Such data, however, is important in case one wishes to train the participants in safety critical situations or simply investigate the possible impact of a specific accident case. Therefore there rises the need for other types of simulation models such as gas dispersion models, fire spreading models etc. Through the integration of such software in the VR system, it is possible for example as soon as a gas leakage occurs, to have a realistic growth of the gas cloud and accurately trigger the on-field gas detectors which in turn will signal certain actions to be performed on behalf of the participants. In failure of the appropriate measures being taken, the gas cloud will expand uncontrollably and in case it reaches a source of high temperature, it could possibly ignite.
Depending on the nature and purpose of the simulation, a number of other assisting software packages could be integrated following the same principle. In order to achieve that, however, it is of paramount importance to ensure during the design phase of the VR system that maintains a modular character and easily allows for cooperation with external software. Such cooperation could be achieved in one of two ways depending on the available computer power as well as whether we wish to maintain a distributed or local character for the VR system. As a first option, the collaborating software could be directly incorporated in the system's codebase in the form of a software library. This solution ensures uninterrupted and latency-free data exchange although significantly raising the amount of required resources by the system. On the opposite side, the external modules could be used as standalone applications distributed in other machines. This requires that a network communication is established between the various modules and the main VR system and interfaces are provided for the data exchange.

\section{Rule-Based Systems}

Up until now, we have discussed the need for external simulators that will realistically implement the behaviour of the virtual environment thus increasing its immersiveness for the participants as well as its credibility towards the safety analysts. However, this environment is static in the sense that the plant's behaviour for example remains the same during different simulation sessions in which many details may differ such as the number of participants, objectives and others. This scenario logic can be implemented through a set of rules that define the consequences when certain criteria are met.

This need can be covered by another external module developed using rule-based technologies. Specifically it is designed to hold an internal set of attributes representing the parameters to monitor. Furthermore it allows the user to import a set of pre-defined rules that specify what actions the system should trigger when specific state changes occur. Therefore, when the VR system reports some change of the monitored attributes, the module checks if a rule applies to the new state and reacts accordingly. The communication of this module with the main VR system follows the principles put out in the previous paragraph. In this case, however, integrating the rule-based module in the main system appears to be the preferred solution as it is expected to be very lightweight in comparison to one of the simulators mentioned above.

\section{Novel Interaction Devices}

It is evident that in such simulation environments as the ones discussed here, time is a key notion. Often, sessions are run simply to measure the time that a 
participant required in order to complete a task or set of tasks. Such measurements, however, are prone to errors due to the simple fact that a virtual environment, regardless how close, never feels $100 \%$ real. Naturally, much effort is being put into reducing such distorting factors to a minimum by increasing the immersiveness of the environment. Towards this goal, a very crucial role is played by the users' interaction devices some of which were shortly presented earlier.

It is important to always keep an eye for new interaction techniques that could possibly enhance the virtual experience. Fairly recently, Nintendo revolutionized the way we interact with video games by offering a novel input device, the Wiimote and Nunchuk, along with its gaming console, the Nintendo Wii. It consists mainly of a "wand-like" joy-pad, the Wiimote, that apart from the usual buttons is enriched with accelerometers and an IR camera. Using two IR light sources, the Wiimote is able to calculate both its position in space as well as its orientation. An additional device accompanying the Wiimote is the Nunchuk which resembles a $3 \mathrm{D}$ mouse bearing two classical buttons and a joystick as well as accelerometers. This is an excellent example of an alternate-purpose device that has lots to offer to virtual reality. It provides tracking functionalities and can be used to recognize hand movements rendering it ideal for tasks such as operating plant components, positioning objects in the environment, issuing hand signals etc. through a more natural and intuitive manner. Furthermore, the classical controls provide an effective means for executing tasks with no real-life counterpart such as controlling a GUI.

\section{Human Factors Technologies}

In every industrial environment, it is humans that form the core of the system's uninterrupted and flawless operation throughout its life-cycle. On the other hand, human actions at either the sharp or the blunt end can become a source of risk. It is therefore mandatory for simulation systems that deal with safety issues to integrate human factors components as an indivisible part of the applied methodologies. Such components will be responsible for assessing the way in which performance variability and deviations may occur as a result of individual actions and thus drive the decision making in regards to developing effective countermeasures.

A VR system, as described until now, provides a controlled environment ideal for collecting the human factors related data required to reach safe decisions. However, it is impractical, and in the case of certain data, impossible to manually record the desired pieces of information. Consequently, it becomes necessary to design the VR system in a way that it is able to automatically store a selected range of relative data. This can be achieved through a logging process able to save a time-stamped history of all notable events such as certain actions of the participants, changes in the properties of environment elements etc.

Although the logging process makes sure that all data needed by human factors technologies (or other simulation data) are collected and stored, they still remain in raw, unprocessed form significantly hindering their direct exploitation. In order to compensate for this problem, the VR system can be coupled with one or more specialized post-processing tools whose goal is to receive the log produced by the simulation and present the desired information directly in human readable form thus automating the process yet more. Further to this, a special-purpose database could be designed as a permanent storage for data collected in past simulation sessions. This history of collected information can be used to produce valuable statistical data as well as provide an indication on the validity of current results thus allowing for example to decide on whether the respective session needs to be repeated. Additionally it gives the safety analysts the opportunity to perform validation tests (through the related sessions) and thus test the efficiency of documented procedures in their real case study by comparing the expected with the actual responses (human, plant, etc).

\section{VR AS A TOOL TO ACHIEVE INDUSTRIAL SAFETY}

There is no doubt that the technological status of Virtual Reality systems has a lot to provide to the general aspect of industrial safety. Presented below are applications of VR systems combining the technological advances towards the aforementioned features implementation and particularly safety related tasks.

\section{VR In Training Simulators}

Virtual Reality simulators can provide realistic behaviour of the training task and the behavioural processes required for the particular task, to fulfil aspects of psychological fidelity as published by Sian \& Robertson, 1999 but also impose the related psychological stress. At the same time such simulators can cope with the aspects of Physical fidelity meaning the degree to which real-world operational equipment is reproduced. This can be provided by advanced VR training simulators being able to model the virtual world (or training environment) as close as possible to reality in order to attain realistic training conditions. They provide thus the ability for the model to behave as the real equipment does and also react to user actions in the same way so that technicians should to be able to perform in the synthetic environment all those relevant actions they would perform in the real world (Stüring, et al, 2002).

Furthermore this kind of simulators can not only provide advanced visualisation in order to represent the simulated environment as close to reality as possible. 
These simulators can also expose the trainee to the full complexity of the task, with not any guidance or feedback at all. The idea of this approach is to foster resourcefulness, problem solving and creativity in the learning process. This could be a very valuable attribute in commissioning, maintenance, or emergency environment where tasks often have to be done for the first time under time or commercial pressure (Stüring, et al, 2002). Additionally, time scaling can also be simulated being an important feature during training activity to help operators familiarizing and getting used with critical and particularly fast events. This can be done with the use of VE systems enabling to adjust reaction times at will of trainees. Other important features that would aim the training session are: zooming/hiding (being able to see things that nobody is allowed to see in reality), colouring (colour pipe paths, routes, flows, etc) and display real-time tags (tag components and their behaviour, especially when combined with process simulators).

\section{VR In Risk Assessment And Accident Investigation}

In this safety action, the great potential usage of VR can be divided into events involving technology-related hazards and events involving human-related hazards. One important aspect is the familiarization with the plant layout and equipment design, and thus the visualization and navigation (walk-through) to enhance analysts' and operators' knowledge on the layout of the plant.

Additionally through a VE simulating an industrial plant, we can have real-time response of fully emulated processes that allow analysts to execute the simulation and analyze options ("what if" scenarios). This can of course be combined with calculation of the volume of liquids inside pipes and vessel and thus enable to see things and processes in a way that is impossible in reality (e.g. volume, type, pressure and temperature of liquids flowing in pipes). This can be done simulated as a whole in a VR system or combined with the usage of an external industrial simulator interfacing to it.

Identification of fire and explosion sources (sparks, friction, etc) can also be of particular importance through a Risk Assessment test case. This can also be simulated with a VR system and provide the necessary information to the safety analyst or even the plant manager so that they take the necessary precautions in case of an explosive gas release or similar situations.

Another feature that VR can provide in relation to technology-related hazards are first of all error detection $\&$ revitalization. Errors can be detected and classified via a VR system by comparing the simulated result with the documented (or expected one). This can be done by combining VR technologies with task execution and monitoring mechanisms and/or rule-based systems. The analysis and actual classification of the error can be done also by post-processing tools (of the data gathered in the VE) and technologies.

Concerning procedures in general inside an industrial environment and specifically the planning stage, a safety analyst can get useful information from a VR simulation by being able to investigate correctness of planned procedures of maintenance (on single parts or the whole plant). Applying the maintenance procedure on virtual components inserted in the system and then evaluating the functionality after the maintenance, one can get feedback directly from the VR system by showing the consequences in the selected point of interest.

\section{VR In Safety Management \& Audit}

The main use of VR technologies are in accident prevention and handling of emergencies, helping safety managers identify possible countermeasures and barriers to ensure that the accident will not happen or minimize its consequences. Using VR, different barriers (that could prevent an accident) can be highlighted or removed during the simulation and thus give the ability to analysts to evaluate their effectiveness analyzing the consequences. These barriers can be physical (eg a wall, door, etc), symbolic (eg lighting, alarm, etc) and functional (eg interlock).

Moreover and under the framework of emergency response and awareness of the situation and communication, VR can help the safety analysts to assess how the orders are adapted in handling emergency procedures. In this way the safety analyst can not only select the suitable emergency team and see the results but also trace different approaches depending on the emergency situation. VR can also offer previewing options imposing thinking of a few options, identifying key personnel to bring new information and finally identifying dangers and problems associated with each option. This way, comparing options abilities are provided in terms of a set of criteria. More analytical comparisons can follow the initial approach in terms of a set of criteria such as, time required to implement options, risks associated with options, resource requirements, allocation and re-allocation of responsibilities and so on.

Another important feature that a VR system can provide is the plant familiarization particularly with the location of emergency exits and other important points. This feature is also related to the training as a safety action as well. One of the most important aspects of evacuation planning is the familiarization of operators with the emergency exits, in normal conditions and especially in altered environment (e.g., fumes and dust in the air). Through VR, the trainee can visualize a path that leads them to the first available emergency exit. The versatility of VR technologies can also allow for creating new hazards during emergency situations 
already envisioned. For instance, operators may have received certain instructions on how to evacuate an area but the evolution of emergency may create addition hazards which must be detected and avoided by the operators while on the move.

Finally close monitoring of works executed inside the plant, compensating for errors and coordinating the work is another feature of particular importance as the ability to monitor the work and the communicating information is a frequent demand for managing emergencies. Virtual reality can facilitate team monitoring and coordination whenever possible to have instantaneous access to all team positions and communications.

\section{PRACTICAL USE CASE}

In this section, we will describe a potential use case that employs a sub-set of the above mentioned technologies in order to enhance the training of new or existing personnel.

The working environment is that of a chemical plant and the targeted personnel are Field Operators (F.Ops) and Control Room Operators (C.R.Ops) who are required to familiarize or gain additional experience with new or existing equipment and procedures. F.Ops are responsible for carrying out tasks on the actual components of the plant such as safety checks or maintenance. On the other hand, C.R.Ops are stationed in a remote control room that allows them to oversee the plant's behaviour and guide the F.Ops in their actions.

A potential and rather frequent scenario would be for a C.R.Op to be alerted by the system for a hazardous situation possibly caused by a malfunction in one of the plant's components. This would trigger a series of protocol events as he/she would have to request from the nearest F.Ops to move into the area and investigate. In turn, the F.Op after approaching the general area would have to act as instructed in order to confirm that the alarm is valid as well as locate its exact source and identify whether the problem is caused indeed by a failure in one of the components. Furthermore, depending on the severity of the situation, a number of steps must be followed so as to safely contain the situation and if possible correct the malfunction. Such a scenario would either end with the problem (temporarily or not) fixed and perhaps some maintenance request issued, or with an escalation of the accident resulting in drastic actions such as plant evacuation and/or emergency shutdown.

Executing training drills based on such a scenario has the obvious benefit that the trainees become accustomed to the emergency processes that must be followed as well as the mechanics of each step. Further to this, however, there is a number of other valuable data to be collected. For example, one can measure reaction times for critical tasks which could lead to reorganising safety procedures or even reassess the ergonomics aspect of the working environment based on observations under time pressure and stress.

On the other hand, holding such drills in the real-life environment is extremely difficult as many of the requested actions interfere with the plant's product flow. This is both highly dangerous in case a human error causes an actual accident but potentially costly as well since interrupting the product flow could be translated in an immediate loss of hundred thousands or even millions of euro.

Consequently, the use of a virtual environment in which such a training session can be reproduced is an ideal solution. In order, however, for it to produce reliable results that can be extrapolated to real life, it is important to provide both an accurate simulation of the actual workspace as well as an immersive user interface.

A VR system designed for such a task would reproduce a realistic $3 \mathrm{D}$ model of the plant's components and surrounding environment in a large screen or projection wall covering the trainees range of sight enabled with stereoscopic vision to enhance the depth perception. The user could control motion with a simple joypad such as the ones used for videogames but also utilise more sophisticated tracked devices (data gloves, head trackers) in order to interact with elements of the environment. In order to guarantee the realistic behaviour of the environment, the virtual environment would further have to be linked to an industrial simulator responsible for calculating in real-time the properties of each of the plant's components. Most industrial sites already use such software products so one needs to build the communication layer between the two software packages. A rules-based system could also be required in order to replicate standard events that cannot be reproduced by the simulator e.g. a worker closing a specific valve when some pressure value reaches a given maximum. Finally, additional components could be needed such as a gas-dispersion model in order to simulate the environmental conditions after an accident has occurred.

Holding training sessions with such a VR installation would produce a wide range of raw data such as the timestamps of a series of events. In order, however, to provide the safety experts with the critical information needed to feed the decision making process, human factors technologies will need to be applied and reform these raw results into high level pieces of information such as the statistics of the occurrence of certain events.

\section{CONCLUSIONS}

In this paper the innovative character of Virtual Reality systems has been presented, having as a main focus the industrial safety. The current status concerning the industrial situation has also been 
presented indicating the main industrial issues currently present in the field and also the missing gaps in the industrial sector. The VR technologies as an innovative technology concerning industrial safety have also been detailed including a draft presentation of the features that could show particular interest in industrial applications and particularly industrial safety. Then the usage of the aforementioned technologies and innovations has been presented including how the application of VR systems could aid particular processes regarding industrial safety.

\section{REFERENCES}

Childs JM, et al. - Handbook of Human Factors Testing and Evaluation, 2002

Gramopadhye, A. K., et al. 2002 "Using Training to Improve Performance of Inspectors on the Hangar Floor", 16th Human Factors in Aviation Maintenance Symposium, April 2-4, 2002.

INTUITION NoE - CRF: "Research Position Paper: Towards Virtual Product Creation: the role of VR in the Automotive \& Transport Industry", 21/02/2006, INTUITION NoE WG2.3.

Jefferson, et al 1995. "Automated hazard identification by emulation of hazard and operability studies", Proceedings of the 8th International Conference on Industrial and Engineering Applications of Artificial Intelligence and Expert Systems. Melbourne, pp765-770, Gordon and Breach Science Publishers.

Loupos K, at al 2007, "VR, HF And Rule-Based Technologies Applied And Combined For Improving Industrial Safety". HCI 2007 Conference Proceedings volume 6, LNCS 4555, ISBN: 978-3-540-73280-9.

Loupos $\bar{K}$. et al. 2007, "Application Of VR And HF Technologies For Improving Industrial Safety", NHIBE conference proceedings, "New Horizons in Industry and Education", 30-31 August 2007, Rhodes Island, Greece.

OSHA - Small Business Handbook, www.osha.gov

Shang X, et al. 2006. "Integrating Vr And Knowledge-Based Technologies To Facilitate The Development Of Operator Training Systems And Scenarios To Improve Process Safety", ESREL 2006 Conference "Safety and Reliability for Managing Risk", 18-22 September 2006, Estoril, Portugal.

Stüring, et al, 2002. "Learning by doing: If at first you don't succeed....", 4th International Conference on New Educational Environments, ICNEE, Lugano, 2002 .

\section{AUTHOR BIOGRAPHIES}

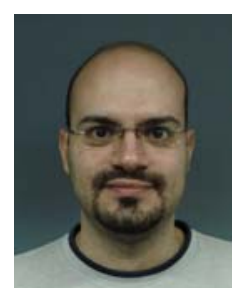

Konstantinos Loupos holds an MSc in Microelectronics Systems Design (University of Southampton UK 2002-2003) with Distinction. His degree thesis was on the setup and programming of an Embedded PC (with $\mathrm{PC} / 104$ interface) for testing of ULAs (Uncommitted Logic Arrays). He also holds an MEng in Electronic And Electrical Engineering (University of Manchester 1998-2002). This latter degree thesis has covered the implementation of a cryptographic algorithm in a Smartcard as well as the design of the related PC (SW) interface and has taken place in Intracom S.A. (Greece). The last four years he has been full-time employed in the Virtual Reality team of the Institute of Communication and Computer Systems (ICCS) in Athens, Greece. His main activities are management and administration of EC projects and also VR development of systems and applications. He has participated in various related conferences with publications as the main author in most of them. His scientific interests range in Electronics, Embedded Systems, Smartcards, and Computer Programming.

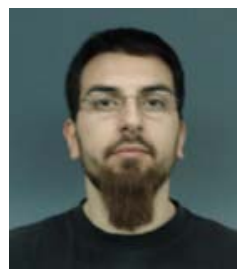

Panagiotis Psonis graduated in 2006 from the Imperial College London (UK) receiving an "M.Sc. in Advanced Computing". The degree thesis involved the research and implementation of contemporary techniques used to enhance realism in computer graphics applications. In 2005 he graduated from the University of Piraeus (Greece) obtaining a "B.Sc. in Computer Science". The final year project required the co-development of a graphical platform that provided the means for comparing AI algorithms. The last two years he has been full-time employed in the Virtual Reality team of the Institute of Communication and Computer Systems (ICCS) in Athens, Greece. His interests lie in the fields of Computer Graphics, Computer Architecture as well as Theory and Design of Programming Languages.

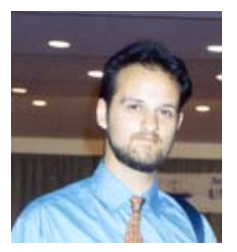

Dr Angelos Amditis has obtained the Diploma in Electrical and Computer Engineering from National Technical University of Athens - NTUA (Greece) in 1992, and a Ph.D. in Electrical and Computer Engineering (Telecommunications) from NTUA (Greece) in 1997. He has been teaching in various courses (communication and computer networks, communication theory etc.) of the Electrical and Computer Engineering Dep. of NTUA and of the Hellenic Naval Academy for the last 5 years. He is employed to ICCS since 1998 and has currently the position of Research Associate Professor. His research interests include model simulation, software and hardware engineering, HMI design and development, Evaluation and consolidation using VE technologies, $\mathrm{VR} / \mathrm{VEs}$ etc. He is scientific responsible in more than 25 European Research projects, 15 of which in the IST area between them and the VIEW project while he was the coordinator of one other 5th Framework Research project. 